\title{
X-Rays through the Looking Glass: Mobile Imaging Dosimetry and Image Quality of Suspected COVID-19 Patients
}

\author{
Alexandra Schelleman', Chris Boyd ${ }^{1,2}$ \\ ${ }^{1}$ South Australia Medical Imaging, Royal Adelaide Hospital, Adelaide, Australia; ${ }^{2}$ Allied Health and Human Performance, University of South Australia, \\ Adelaide, Australia
}

\section{Technical Paper}

Received December 10, 2020

Revision March 19, 2021

Accepted May 27, 2021

Corresponding author:

Alexandra Schelleman

South Australia Medical Imaging, Royal Adelaide Hospital, Port Road,

Adelaide 5000, Australia

E-mail: alex.schelleman@sa.gov.au

(iD https://orcid.org/0000-0002-4677-4285

This is an open-access article distributed under the terms of the Creative Commons Attribution License (http://creativecommons.org/licenses/by-nc/4.0), which permits unrestricted use, distribution, and reproduction in any medium, provided the original work is properly cited.

\section{Copyright $\odot 2021$ The Korean Association for} Radiation Protection

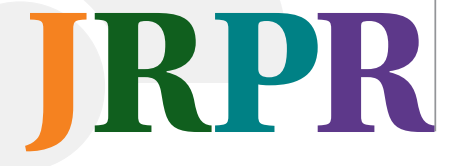

Background: This paper aims to evaluate the clinical utility and radiation dosimetry, for the mobile X-ray imaging of patients with known or suspected infectious diseases, through the window of an isolation room. The suitability of this technique for imaging coronavirus disease 2019 (COVID-19) patients is of particular focus here, although it is expected to have equal relevance to many infectious respiratory disease outbreaks.

Materials and Methods: Two exposure levels were examined, a "typical" mobile exposure of $100 \mathrm{kVp} / 1.6 \mathrm{mAs}$ and a "high" exposure of $120 \mathrm{kVp} / 5 \mathrm{mAs}$. Exposures of an anthropomorphic phantom were made, with and without a glass window present in the beam. The resultant phantom images were provided to experienced radiographers for image quality evaluation, using a Likert scale to rate the anatomical structure visibility.

Results and Discussion: The incident air kerma doubled using the high exposure technique, from $29.47 \mu \mathrm{Gy}$ to $67.82 \mu \mathrm{Gy}$ and scattered radiation inside and outside the room increased. Despite an increase in beam energy, high exposure technique images received higher image quality scores than images acquired using lower exposure settings.

Conclusion: Increased scattered radiation was very low and can be further mitigated by ensuring surrounding staff are appropriately distanced from both the patient and X-ray tube. Although an increase in incident air kerma was observed, practical advantages in infection control and personal protective equipment conservation were identified. Sites are encouraged to consider the use of this technique where appropriate, following the completion of standard justification practices.

Keywords: Mobile X-ray, Infection Control, COVID-19, Dose

\section{Introduction}

Healthcare associated infection is the most frequent adverse event in healthcare [1], resulting in longer hospital admissions, higher costs, and unnecessary deaths of patients and health care workers. In recent years, outbreaks of highly infectious diseases such as Ebola virus, Middle East respiratory syndrome (MERS) and coronavirus disease 2019 (COVID-19), have emphasized the importance of infection control measures for the protection of patients and staff.

The spread of COVID-19 is slowed by infection control measures, such as hand and respiratory hygiene, waste management and the use of personal protective equipment 
(PPE) [2]. The current World Health Organization (WHO) guidelines advises droplet precautions be observed for suspected or confirmed COVID-19 patients, unless an aerosolgenerating procedure (e.g., intubation, bronchoscopy) is being performed, where an N95 mask is recommended [2]. Due to increased demand, long-term issues have arisen with the supply of N95 masks and other medical equipment essential to the management of COVID-19 [3]. As the virus causing COVID-19 has been detected on plastic and steel up to 72 hours post application [4], X-ray tubes, tables and other medical equipment must be thoroughly cleaned and disinfected [2]. The WHO therefore recommends the use of dedicated portable X-ray equipment for imaging of COVID-19 patients [2].

Our hospital is a major trauma center with a 70-bed emergency department (ED) and 60-bed intensive care unit (ICU). The majority of ED patient rooms have a glass door allowing observation by ED staff. Where possible, suspected or confirmed COVID-19 patients have been imaged with the digital radiography (DR) unit outside of the room, performing the exposure "through the glass." This technique has been previously described by Mossa-Basha et al. [5] and Brady et al. [6] with the following advantages:

- Decreased time required for each patient examination, as cleaning of the mobile DR unit is reduced between patients.

- Reduced risk of mobile DR unit contamination.

- Halving PPE consumption per examination, as only 1 radiographer is required to put on (or "don") PPE, rather than 2.

This technique exploits the glass-fronted room design often present in the hospital environments. Making an X-ray exposure through glass allows radiographers to see the patient and position the X-ray unit appropriately, whilst maintaining a physical barrier between the infectious patient and the machine. In order to compensate for the increased source to image-receptor distance (SID), as well as the presence of the glass in the primary beam, a higher exposure technique is used.

Whilst this technique provides some advantages, there are potential disadvantages such as an increase in radiation exposure due to scattered radiation and a potential for reduced image quality. This study simulates both acquisition methods for the imaging of COVID-19 patients, measuring the dose to surrounding staff and examining the impact on image quality.
This study was undertaken to critically evaluate the appropriateness of this technique in a clinical setting. The study objectives were twofold, seeking to quantify scattered radiation doses from a modified "through the glass" technique, and address whether image quality is reduced by glass attenuation or increased exposure factors.

\section{Materials and Methods}

\section{Shooting "Through the Glass"}

The clinical protocol for chest X-ray exposures "through the glass" is as follows:

(1) Radiographers take the mobile machine to the patient room.

(2) The digital cassette (without grid) is "double bagged" in two disposable plastic covers to ensure that no edges are exposed to the patient environment

(3) Typically used peak tube kilovoltage $(\mathrm{kVp})$ and current-time (mAs) values of $100 \mathrm{kVp}$ and $1.6 \mathrm{mAs}$ are modified to $120 \mathrm{kVp}$ and $5 \mathrm{mAs}$.

(4) Radiographer_1 dons PPE and enters patient room with digital detector.

(5) Radiographer_1 identifies and positions patient.

(i) Antero-posterior (AP) erect: patient is erect on bed facing window, the detector placed behind their back.

(ii) Postero-anterior (PA) erect: patient is stood erect with their back towards the window, holding the detector to front of chest.

(6) Radiographer_2 positions mobile DR unit as close to the glass as possible, communicating with Radiographer_1 using improvised hand signals. Light in patient room is turned off to aid visualization of the light field on patient anatomy.

(7) Prior to exposure, Radiographer_1 moves to the corner of the room and gives the patient breathing instructions.

(8) Following exposure, Radiographer_2 confirms image is adequate using hand signals.

(10) Radiographer 1 takes the detector from patient and passes the detector to Radiographer_2, retaining the outer disposable plastic cover.

(11) Radiographer_1 removes (or 'doffs') PPE before leaving the patient room, while Radiographer_2 removes the second disposable cover, cleaning the detector surface with isopropyl alcohol wipes. 
(12) Radiographer_2 labels image and completes examination, including image annotation for reporting a radiologist, indicating image was acquired through glass.

\section{Experimental Design}

In order to mimic the conditions described above, a low
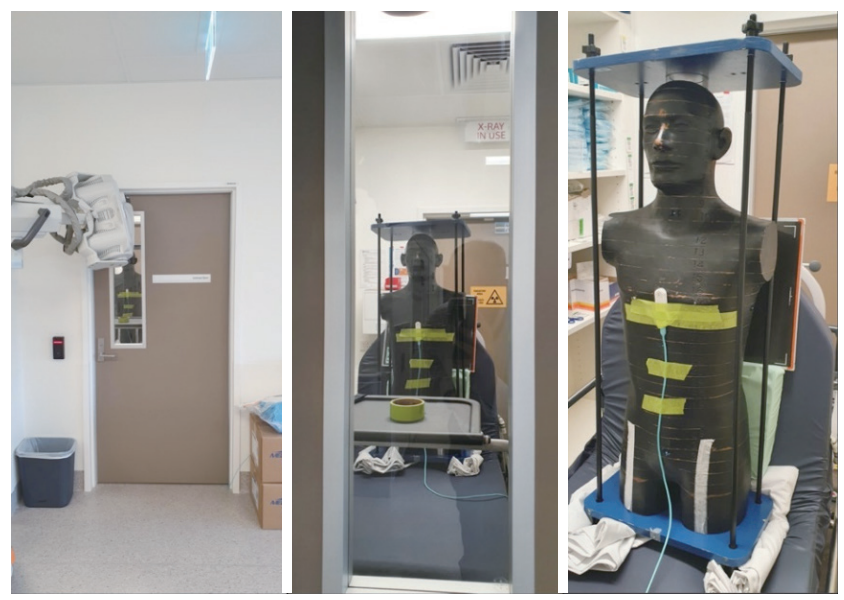

Fig. 1. Photographs showing (left to right) tube location outside room, phantom as viewed through window, phantom with dosimeter at sternum. traffic room with a glass window was found in the Radiology department and shown in Fig. 1. The glass was standard household glass, with a thickness of around $5 \mathrm{~mm}$. A RANDO anthropomorphic phantom (Phantom Laboratory, Salem, NY, USA) was placed on an ED bed inside the room with the DR detector placed behind the phantom. This phantom is composed of varying polymer densities, designed to mimic the attenuation and scatter of real human tissue.

A Carestream DRX-Revolution mobile DR unit (Carestream Health, Rochester, NY, USA) was positioned outside the room at a SID of $219 \mathrm{~cm}$ to mimic the SID achieved clinically. The source to glass distance, where applicable, was $49 \mathrm{~cm}$ and full experimental setup is shown in Fig. 2. Absorbed dose, peak dose rate, half value layer (HVL) and exposure duration were measured using a RaySafe X2 solid state radiation dosimetry system (RaySafe, Billdal, Sweden).

The dosimeter location, glass presence and exposure parameters were used in different combinations, creating seven unique images listed in Table 1 . The exposures tested were $100 \mathrm{kVp} / 1.4 \mathrm{mAs}$ (a small patient exposure without glass present), $100 \mathrm{kVp} / 1.6 \mathrm{mAs}$ (a typical patient exposure without glass present) and $120 \mathrm{kVp} / 5 \mathrm{mAs}$ (exposure setting modified for "through the glass" examinations). Combinations of these

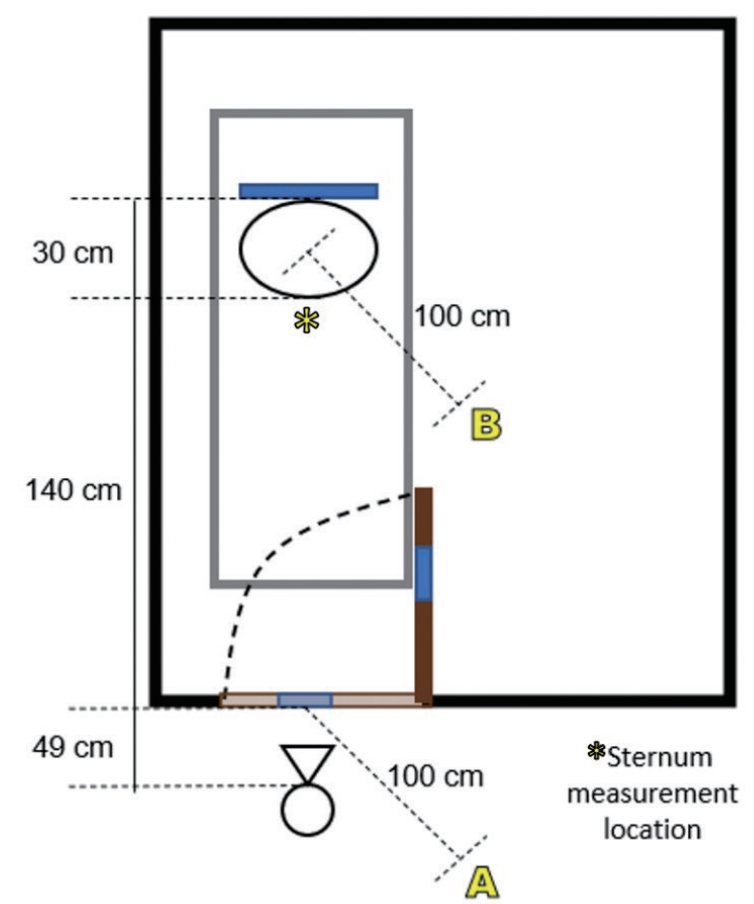

A - dosimeter measuring scatter radiation $100 \mathrm{~cm}$ from glass window, to mimic location of emergency department staff

D - dosimeter measuring scatter radiation $100 \mathrm{~cm}$ from the phantom, to mimic location of radiographer within patient room

Fig. 2. Experimental set-up with dosimeter locations marked. 
Table 1. Experimental Set-up and Exposure Settings for Each Image

\begin{tabular}{lcccl}
\hline $\begin{array}{l}\text { Image } \\
\text { number }\end{array}$ & $\begin{array}{c}\text { Peak tube } \\
\text { voltage } \\
(\mathrm{kVp})\end{array}$ & $\begin{array}{c}\text { Tube } \\
\text { time-current } \\
\text { product (mAs) }\end{array}$ & $\begin{array}{c}\text { Presence of } \\
\text { glass in } \\
\text { exposure }\end{array}$ & $\begin{array}{c}\text { Dosimeter } \\
\text { position }\end{array}$ \\
\hline 1 & 100 & 1.4 & Yes & Sternum \\
2 & 120 & 5 & Yes & Scatter in room \\
3 & 100 & 1.6 & No & Sternum \\
4 & 100 & 1.6 & Yes & Sternum \\
5 & 120 & 5 & No & Scatter in room \\
6 & 100 & 1.6 & No & Sternum \\
7 & 100 & 1.6 & Yes & Sternum \\
\hline
\end{tabular}

exposure settings were used both with and without glass present. The modified "through the glass" exposure was chosen based on a review of the limited body of existing literature, and discussion with radiographers using this technique at other clinical centers.

\section{Image Quality Evaluation}

There was concern expressed by radiographers that performing imaging through the glass would produce poor quality and potentially non-diagnostic imaging, even with a higher exposure technique. However, the objective image quality evaluation is a notoriously difficult task. In order to assess the quality of the images obtained here, a survey was created based upon the Royal Australian and New Zealand College of Radiologists (RANZCR) CT Image Review Self-Audit [7] and the European Guidelines on Quality Criteria for Diagnostic Radiographic Images [8].

Images were made available to the clinical staff via the hospital picture archive and communication system (PACS), numbered from one to eight. All identifying exposure information, including relevant DICOM (Digital Imaging and Communications in Medicine) header information, was removed to blind reviewers and ensure objectivity.

Images were rated from one (poorly represented/unseen) to five (visually sharp) and the survey was completed by radiographers ranging in experience from 7 to 20 years. Scoring was based on the criteria listed below, with the phantom images shown in Fig. 3:

- Sharpness of tracheal wall at the level of the carina

- Soft tissue representation

- Bony trabeculae of clavicles

- Vertebral plates seen through mediastinum/upper abdomen

- Reproduction of costophrenic angles
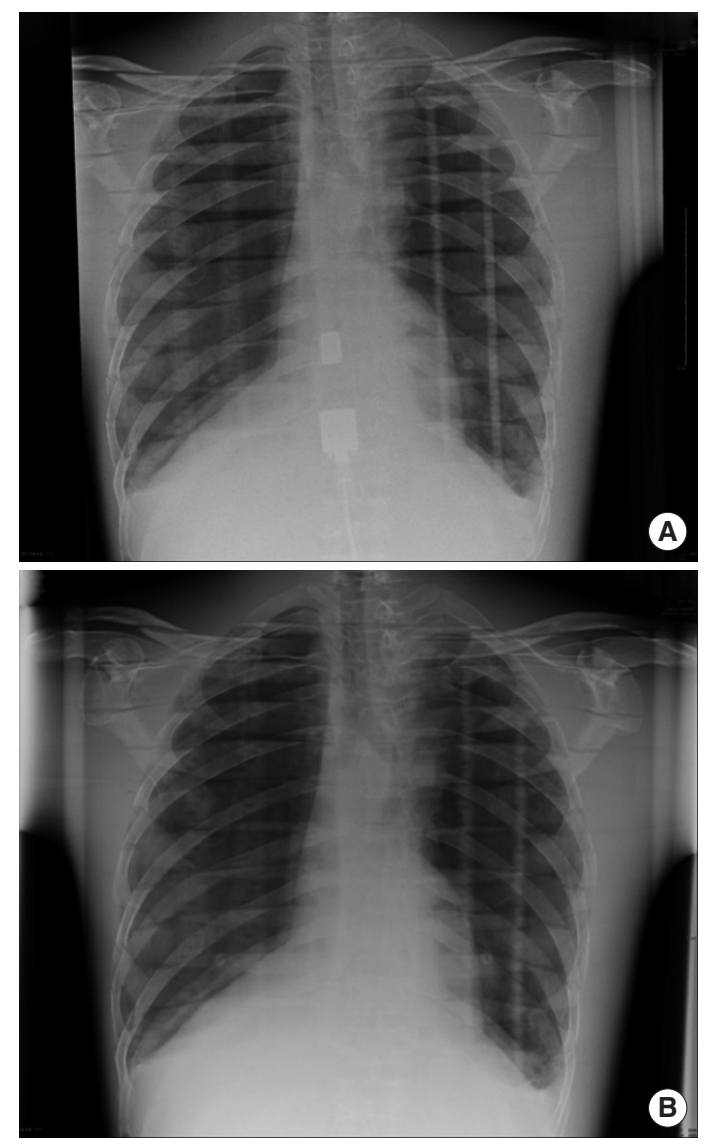

Fig. 3. Example of phantom images taken at (A) $100 \mathrm{kVp} / 1.4 \mathrm{mAs} /$ glass and (B) $120 \mathrm{kVp} / 5 \mathrm{mAs} / \mathrm{no}$ glass.

Assessors were also asked to provide a wholistic assessment of clinical image quality suitability and what (if any) modifications to exposure settings would be suggested. One image was also included twice, to assess intra-observer reliability.

The five scoring criteria listed above were then totaled to provide a quality weighted average mark (WAM) out of 25 , for each image, as per Equation (1).

Quality $W A M=\bar{x}($ quality criteria 1$)$

$$
+\bar{x} \text { (quality criteria } 2) \ldots \bar{x} \text { (quality criteria } 5 \text { ) }
$$

\section{Results and Discussion}

\section{Dose Results}

Radiation dosimetry results are summarized in Table 2, showing the impact of the $120 \mathrm{kVp} / 5 \mathrm{mAs}$ technique. As expected, instantaneous dose rate and cumulative dose at the scattered measurement locations was increased when higher exposure settings were used. In all instances however, 
Table 2. Measured Doses at Sternum, Location A and Location B (standard error shown to 1 significant figure)

\begin{tabular}{lccccccc}
\hline $\begin{array}{c}\text { Peak tube } \\
\text { volatage }(\mathrm{kVp})\end{array}$ & $\begin{array}{c}\text { Tube time-current } \\
\text { product }(\mathrm{mAs})\end{array}$ & $\begin{array}{c}\text { Measurement } \\
\text { position }\end{array}$ & $\begin{array}{c}\text { Dose } \\
(\mu \mathrm{Gy})\end{array}$ & $\begin{array}{c}\text { Peak dose rate } \\
(\mathrm{mSV} / \mathrm{min})\end{array}$ & $\begin{array}{c}\mathrm{HVL} \\
(\mathrm{mmAl})\end{array}$ & $\begin{array}{c}\text { Exposure time } \\
(\mu \mathrm{s})\end{array}$ & $\begin{array}{c}\text { Glass } \\
\text { present }\end{array}$ \\
\hline 100 & 1.6 & Sternum & $13.25 \pm 0.08$ & $123.96 \pm 0.24$ & 5.886 & $6.21 \pm 0.02$ & Yes \\
100 & 1.6 & Sternum & $29.47 \pm 0.10$ & $272.08 \pm 1.20$ & 3.818 & $6.50 \pm 0.04$ & No \\
100 & 1.6 & $\mathrm{~A}$ & $0.20 \pm 0.001$ & $328.45 \pm 5.400$ & - & - & Yes \\
100 & 1.6 & $\mathrm{~B}$ & $0.07 \pm 0.0005$ & $111.28 \pm 2.4000$ & - & No \\
120 & 5 & Sternum & $67.82 \pm 0.0003$ & $203.60 \pm 1.2000$ & 6.825 & $20.13 \pm 0.03$ & Yes \\
120 & 5 & $\mathrm{~A}$ & $0.99 \pm 0.11$ & $1,571.76 \pm 0.48$ & - & - & Yes \\
120 & 5 & $\mathrm{~B}$ & $0.51 \pm 0.001$ & $817.80 \pm 0.600$ & - & - & Yes \\
120 & 5 & $\mathrm{~A}$ & $1.38 \pm 0.002$ & $2,190.20 \pm 6.000$ & - & No \\
\hline
\end{tabular}

$\mathrm{HVL}$, half value layer.

Table 3. Radiographer Evaluated Image Quality

\begin{tabular}{|c|c|c|c|c|c|c|c|c|}
\hline \multirow{2}{*}{ Image \# } & \multirow{2}{*}{$\begin{array}{c}\text { Peak tube } \\
\text { voltage (kVp) }\end{array}$} & \multirow{2}{*}{$\begin{array}{l}\text { Tube time-current } \\
\text { product (mAs) }\end{array}$} & \multirow{2}{*}{$\begin{array}{l}\text { Glass } \\
\text { present }\end{array}$} & \multicolumn{3}{|c|}{ Image based exposure optimisation recommendation } & \multirow{2}{*}{$\begin{array}{l}\text { Quality } \\
\text { WAM }\end{array}$} & \multirow{2}{*}{$\begin{array}{l}\text { Dose area product } \\
\text { of X-ray }\left(\mathrm{dGy} \cdot \mathrm{cm}^{2}\right)\end{array}$} \\
\hline & & & & Increase & Decrease & Adequate & & \\
\hline 1 & 100 & 1.4 & Yes & $4 / 5$ & - & $1 / 5$ & 14.30 & 0.256 \\
\hline 2 & 120 & 5 & Yes & - & - & $1 / 5$ & 20.20 & 1.698 \\
\hline 3 & 100 & 1.6 & No & - & - & $1 / 5$ & 17.50 & 0.385 \\
\hline 4 & 100 & 1.6 & Yes & $3 / 5$ & - & $2 / 5$ & 17.40 & 0.385 \\
\hline 5 & 120 & 5 & No & $1 / 5$ & $2 / 5$ & $2 / 5$ & 21.60 & 1.698 \\
\hline 6 & 100 & 1.6 & No & - & - & $1 / 5$ & 19.05 & 0.385 \\
\hline 7 & 100 & 1.6 & Yes & - & - & $1 / 5$ & 18.85 & 0.384 \\
\hline 8 & 120 & 5 & Yes & - & $2 / 5$ & $3 / 5$ & 21.60 & 1.703 \\
\hline
\end{tabular}

WAM, weighted average mark.

doses remained below $1 \mu \mathrm{Gy}$ for cumulative scattered radiation measurements. An increased dose rate without glass was seen due to changes in exposure duration. This is shown by the increased cumulative sternal dose for exposures performed with glass, despite the lower dose rate.

\section{Image Quality Results}

Five experienced radiographers (mean years qualified $=$ 13.25) evaluated the phantom images. The image quality criteria described above were evaluated on a Likert scale between 1 and 5, allowing an average score to be evaluated for each quality criteria.

As shown in Table 3, image \#1 (100 kVp/1.4 mAs/glass) was regarded as the poorest quality image, with $4 / 5$ respondents stating that they would increase the exposure if repeating. This image also scored the lowest WAM across the five image quality measures, and was ranked the worst overall by $3 / 5$ of the radiographers. Images \#4 and \#7 (100 kVp/1.6 mAs/glass) also ranked lower overall and had low quality WAMs. The best performing image, across both WAM and overall ranking was image \#5 (120 kVp/5 mAs/no glass), although this image was considered overexposed by $2 / 5$ respondents. The images obtained using a higher exposure technique (120 kVp/5 mAs) had higher WAMs and rankings than images obtained at lower exposures.

\section{Dose Discussion}

This study was performed to quantify radiation dose to both the patient and the surrounding medical staff by the use of a higher exposure, "through the glass" technique. The incident air kerma to the phantom using $120 \mathrm{kVp} / 5 \mathrm{mAs} /$ glass $(67.82 \mu \mathrm{Gy})$ was double that of the "normal" mobile $\mathrm{X}$ ray exposure at $100 \mathrm{kVp} / 1.6 \mathrm{mAs} /$ no glass (29.47 $\mu \mathrm{Gy})$. However, both of these values remain below the $160 \mu \mathrm{Gy}$ diagnostic reference level for PA chest X-rays, published elsewhere [9]. While the high exposure technique produces a higher incident air kerma, it also:

- Decreases the amount of time taken to perform chest $\mathrm{X}$-rays on patients with suspected COVID-19 or other infectious disease, due to reduced equipment cleaning.

- Decreases the infection risk of taking a mobile machine between "dirty" rooms.

- Halves the amount of radiographers needed in the room, saving PPE. 
By measuring dose at the "sternum" of the phantom, a HVL was calculated for glass across the tested exposures. At $100 \mathrm{kVp} / 1.6 \mathrm{mAs}$, the HVL was $5.89 \mathrm{mmAl}$ (aluminum), whilst at $120 \mathrm{kVp} / 5 \mathrm{mAs}$, the HVL was $6.83 \mathrm{mmAl}$. This is consistent with the findings of Brady et al. [6], who tested six different types of glass and found that the HVL for X-rays at $120 \mathrm{kVp}$ ranged between 6.0 and $7.1 \mathrm{mmAl}$. For this reason, Brady et al. [6] recommend a "through glass" technique using 110$120 \mathrm{kV}$, with a mAs value of four times the standard mobile chest $\mathrm{X}$-ray value, assuming a distance from tube to detector of approximately $2 \mathrm{~m}$. These recommendations are consistent with the results seen here for a "through glass" exposure of $120 \mathrm{kVp}$ and $5 \mathrm{mAs}$, given the local standard AP chest Xray exposure of 1-1.6 mAs.

One of the concerns expressed by ED staff was the amount of scattered radiation produced from the primary beam incident on glass. The measured radiation at position A ( $1 \mathrm{~m}$ away from the glass outside the room) was $0.99 \mu \mathrm{Gy}$ using the higher exposure technique. This dose is very low and does not place surrounding staff at risk if standard radiation protection principles are adhered to. Similar measurements by Brady et al. [6] found that, at $1 \mathrm{~m}$ from the glass outside the room, the dose was less than $0.5 \mu \mathrm{Sv}$. To contextualize these results, a person would need to be exposed to this scatter 2,000 times to exceed the $1 \mathrm{mSv}$ Australian public dose limit. In practice, it is rare that individuals stand as close as $1 \mathrm{~m}$ from the glass, meaning that the exposure of the radiographer and ED staff outside these rooms would be even smaller as a result of the inverse square law. This dose would be further reduced by adhering to normal radiation practices, such as:

- Clearly stating "X-ray!" immediately prior to an exposure being made.

- Asking emergency department staff to maintain a distance of more than $2 \mathrm{~m}$ during exposure (reducing the dose to one quarter of that measured at $1 \mathrm{~m}$ ).

The scatter at position B (1 m from the phantom inside the room) was found to be $0.07 \mu \mathrm{Gy}$ for the low exposure technique and $0.05 \mu \mathrm{Gy}$ for the higher exposure technique. Radiographers routinely wear lead under PPE, to reduce radiation exposure when remaining in the room with the patient. As mentioned above, this exposure is very low, even without the addition of lead.

\section{Image Quality Discussion}

The above dose results are irrelevant if this technique compromises image quality. In order to assess image quality, im- age analysis was conducted by a number of experienced radiographers. As expected, image \#1 (100 kVp/1.4 mAs/glass) was scored the lowest by respondents. This exposure would be routine for patient imaging "without" the presence of glass and at a shorter SID. It would be expected that the addition of glass results in reduced image quality for these radiographs, as is supported by the low WAM for these images.

Images scoring the highest WAM were 2, 5, and 8, all of which were obtained using the higher exposure technique. Images \#2 and \#8 were taken through glass, while image \#5 was taken without glass. This indicates phantom anatomical landmarks were better visualized using the higher exposure technique. Interestingly, some radiographers indicated that they would decrease the exposure setting for images \#5 and \#8 if repeating. These data support the use of this technique for the production of diagnostic chest X-ray radiographs.

In practice, chest $\mathrm{X}$-rays acquired using this technique were sent to radiologists with a note stating that they had been acquired through glass. Anecdotally, feedback from radiologists about this technique was positive, with radiographers also preferring to use this technique wherever possible. Radiographers are required to assess image quality at the time of exposure to determine whether imaging is of diagnostic quality, and if the "through the glass" technique did not produce diagnostic imaging, radiographers could revert to the standard procedure of entering the room with the mobile X-ray machine.

\section{Conclusion}

This study was designed as a test of the existing clinical protocol, which results in several limitations. This study analysed a limited set of exposure values. Although not examined here, an intermediary exposure (e.g., $110 \mathrm{kVp} / 3 \mathrm{mAs}$ ) may produce adequately exposed images with lower incident air kerma and could be examined in future work.

Analysis of anthropomorphic phantom images was also challenging, due to the lack of "real" anatomy. For example, the "bronchial tree" of the phantom (seen in Fig. 3) did not extend far past the carina. Typical image quality criteria such as rotation, projectional correctness and inspiration were not applicable. Brady et al. [6] conducted a similar analysis of images obtained "through the glass" of hospital rooms, but analyzed radiographs obtained in clinical practice. Their thorough analysis included criteria such as "mandible is not in the exposure field," unfortunately not applicable to our rigid 
phantom. Even with this limitation however, our assessing radiographers consistently scored the higher exposure "through glass" images better than lower exposure images.

In conclusion, highly infectious and/or airborne diseases present both radiographers and the wider health community with a slew of challenges. For many of these diseases, mobile $\mathrm{X}$-ray imaging has shown utility in both initial and ongoing evaluation. In the particular case of COVID-19 management, this study has shown that ED patients presenting with suspected or confirmed COVID-19 could be imaged through glass with high image quality. By using the "through the glass" technique described above, the risk of transmitting infectious diseases on the surface of the machine is almost entirely eliminated. Additionally, the number of staff required to don PPE and enter the patient room is halved, saving both valuable resources and reducing the risk to staff themselves. This study demonstrated that a higher exposure technique did result in a higher incident air kerma, but also resulted in favourable results for image quality. While the scattered radiation is increased both inside and outside the glass, the increase is negligible and the risk to the surrounding staff can be mitigated by using standard radiation protection practices such as ensuring sufficient distance of any staff from the tube and patient.

\section{Conflict of Interest}

No potential conflict of interest relevant to this article was reported.

\section{Author Contribution}

Conceptualization: Schelleman A, Boyd C. Investigation and methodology: Schelleman A. Resources: Boyd C. Writing of the original draft: Schelleman A. Writing of the review and editing: Schelleman A, Boyd C. Formal Analysis: Boyd C.

\section{References}

1. World Health Organization. The Burden of Health Care-Associated Infection Worldwide [Internet]. Geneva, Switzerland: World Health Organization; 2010 [cited 2021 Aug 27]. Available from: https://www.who.int/gpsc/country_work/summary_20100430_ en.pdf.

2. World Health Organization. Infection prevention and control during health care when novel coronavirus (nCoV) infection is suspected [Internet]. Geneva, Switzerland: World Health Organization; 2020 [cited 2021 Aug 27]. Available from: https://www. who.int/publications/i/item/10665-331495.

3. World Health Organization. Rational use of personal protective equipment (PPE) for coronavirus disease (COVID-19) [Internet]. Geneva, Switzerland: World Health Organization; 2020 [cited 2021 Aug 27]. Available from: https://www.who.int/publications/i/item/rational-use-of-personal-protective-equipment(ppe)-for-coronavirus-disease-(covid-19).

4. van Doremalen N, Bushmaker T, Morris DH, Holbrook MG, Gamble A, Williamson BN, et al. Aerosol and surface stability of SARSCoV-2 as compared with SARS-CoV-1. N Engl J Med. 2020;382(16): 1564-1567.

5. Mossa-Basha M, Medverd J, Linnau KF, Lynch JB, Wener MH, Kicska G, et al. Policies and guidelines for COVID-19 preparedness: experiences from the University of Washington. Radiology. 2020;296(2):E26-E31.

6. Brady Z, Scoullar H, Grinsted B, Ewert K, Kavnoudias H, Jarema A, et al. Technique, radiation safety and image quality for chest $\mathrm{X}$-ray imaging through glass and in mobile settings during the COVID-19 pandemic. Phys Eng Sci Med. 2020;43(3):765-779.

7. Royal Australian and New Zealand College of Radiologists. CT Image Review Self-Audit Worksheet [Internet]. Sydney, Australia: Royal Australian and New Zealand College of Radiologists; c2021 [cited 2021 Aug 27]. Available from: https://www.ranzcr. com/college/document-library/ct-image-review-self-auditworksheet.

8. European Commission. European guidelines on quality criteria for diagnostic radiographic images. Luxembourg: European Commission; 1996.

9. Hart D, Hillier MC, Shrimpton PC. Doses to patients from radiographic and fluoroscopic X-ray imaging procedures in the UK: 2010 review (HPA-CRCE-034). Didcot, UK: Health Protection Agency; 2012. 